\title{
THE STUDY OF NONLINEAR EFFECTS INFLUENCED BY SPACE CHARGE IN HIGH INTENSITY LINAC
}

\author{
A. A. Kolomiets, S. G. Yaramishev, P. R. Zenkevich, A. P. Korolev
}

Institute for Theoretical and Experimental Physics, Moscow, Russia

\section{INTRODUCTION}

Beam halo formation in high intensity linacs is now extensively studied due to the problem of particle losses is a critical condition for design of high current $\mathrm{cw}$ linacs for nuclear waste transmutation and power production facilities. The beam losses less than $1 \mathrm{nA} / \mathrm{m}$ for high energy part of linac are considered now as acceptable level. It is clear that requirement of such low level losses leads either to beam current limitation or such set of linac parameters, which can considerably decrease its efficiency.

As it follows from the previous and recently carried out investigations the main reasons of the beam halo formation are nonlinear effects. The latest review of these works is given in [1]. These effects have their origin in influence of the space charge forces, mismatched initial distribution, nonlinear external focusing, etc. In many cases as this takes place Hamiltonian becomes time dependent:

$$
H=H_{0}(I)+\varepsilon V(I, \theta, t)
$$

where $H_{0}$ - Hamiltonian of nonperturbed system, $\varepsilon$ perturbation parameter, $V(I, \theta, t)$-time periodic function, $I, \theta$ action - angle variables, $t$ - time.

This leads to appearance of nonlinear resonances which makes beam dynamics much more complicated. The simulations show that for rather big perturbation parameter (high beam current for example) this is reflected in the fact that beam emittance grows. Nevertheless there is certain range of perturbation for which simulations do not show any emittance growth but appearance of the beam halo is experimentally observed.

It is known [2] that even for very small perturbation parameter there exist stochastic trajectories which correspond to the destroyed tori whose measure, however, is exponentially small. To clear up the low particle losses problem it is necessary to investigate the beam dynamics at rather small perturbation. In present paper the using of the correlation function for analysis of beam dynamics simulation results is discussed.

\section{SPECTRAL PROPERTIES OF DYNAMICAL SYSTEMS}

The consideration of some specific spectral properties of dynamical systems in the transition region from order to chaos is given in [3]. In this work the simplest map that arises in the nonlinear oscillation theory has been studied:

$$
\begin{aligned}
I_{n+1} & =I_{n}+K \sin \vartheta_{n}, \\
\vartheta_{n+1} & =\vartheta_{n}+I_{n+1} \quad \bmod 2 \pi,
\end{aligned}
$$

where $K$ is parameter of the problem and $n$ is discrete time.
For some sequence $x_{k}=f\left(I_{k}, \vartheta_{k}\right)$ the spectral density of the correlation function is determined by the expression

$$
\begin{aligned}
& R_{N}\left(\omega_{m}\right)=\frac{1}{N} \sum_{-\frac{1}{2} N \leq k \leq \frac{1}{2} N} R_{N}(k) e^{-i k \omega_{m}} \\
& R_{N}(k)=\frac{1}{N} \sum_{-\frac{1}{2} N \leq j \leq \frac{1}{2} N} x_{j} x_{j+k}, \quad \omega_{m}=2 \pi \frac{m}{N}
\end{aligned}
$$

In this expression $N$ determines the maximum of map steps.

It follows from given in [3] qualitative analysis of spectrum of correlation function that it is determined by structure of the phase space. Inside it one can see the stability islands which correspond to the resonances of different orders. They point out in spectrum for $K<<1$ the local maxima with corresponding resonant frequencies. As $K$ increases the spectrum becomes wider and nearly uniform.

There is one more universal spectral property of mapping (2). Well marked peak appears for zero frequency when $K \approx 1$ (so called "central peak"). It is due to some trajectories pass the vicinity of hyperbolic point of the separatrix for a very long time. This peak is a good indicator of stochastic motion region appearance.

\section{ANALYSIS OF THE BEAM DYNAMICS SIMULATION RESULTS}

Let us consider now spectral properties of particle trajectories in periodic focusing channels. It is well known that trajectory is defined by expression

$$
\begin{aligned}
& x=\sqrt{\frac{2 I_{x} L}{\varepsilon_{x}}}\left|\varphi_{x}\right| \cos \left(\mu \tau+\theta_{x}\right) \\
& I_{x}=\frac{2 \varepsilon_{x}\left|\tilde{c}_{x}\right|^{2}}{L} ; \quad \theta_{x}=\arg \tilde{c}_{x},
\end{aligned}
$$

where $\tilde{c}_{x}$ - complex amplitude of oscillation, $L$ - length of focusing period, $\varphi_{x}$ - Flouqet function, which depends on Coulomb parameter $h$ and on type of initial particle distribution in phase space, $\mu$ - phase advance of Flouqet function on the focusing period, $\varepsilon_{x}$ - emittance in corresponding plane and $\tau$ - dimensionless time.

Coulomb parameter depends on phase density of beam current and on focusing channel parameters [4]:

$$
h=\frac{\lambda k}{\mu_{0} \beta \gamma^{2} J_{0}} \cdot \frac{J}{\varepsilon_{x, y}} \text {. }
$$

here $\lambda$ - wave length of accelerating field, $k=\frac{L}{\beta \lambda}, J$ - beam current, $\beta, \gamma$-relativistic parameters, $\mu_{0}$ - phase advance for 
$J=0, J_{0}$ - characteristic current.

It is shown in [] that dependence of phase advance $\mu$ on Coulomb parameter is expressed by

$$
\mu=\mu_{0}\left(\sqrt{1+h^{2}}-h\right)
$$

In [5] the influence of structural resonances in periodic focusing structures on beam dynamics are considered. In this work the analytical study has been carried out for smooth approximation and for uniform distribution of particles in phase space. Following [5] the Hamiltonian in the vicinity of resonance with number $k$ can be determined by expression

$H=\frac{2 \pi I_{\max }}{L}\left[\sum_{n=1} \frac{v_{n}}{n} \tilde{I}^{n}+\frac{g_{k}}{k} \tilde{I}^{k} \cos \left[2 k\left(2 \pi \delta \tau+\theta_{x}\right)\right]\right]$

where $\delta=\frac{\mu}{2 \pi}-\frac{1}{2 k}, \tilde{I}=\frac{I_{x}}{I_{\max }}, g_{k}$-depended on $h$ width of the $k$-th resonance, $v_{1}$ - linear shift of the transverse oscillation frequency, $v_{n>1}$ - nonlinear frequency shift for particles with maximum amplitude influenced by $2 n$-th nonlinearity of potential $V(I, \vartheta, t)$.

Following the procedure given in [2] one can write the mapping for (7):

$\vartheta_{n+1}=\vartheta_{n}+2 \pi\left(v_{1}+v_{2} \tilde{I}_{n}\right)+g_{k} 2 \pi \tilde{I}_{n}{ }^{k} \cos \left[2 k\left(2 \pi n \delta-\theta_{n}\right)\right]$

$\tilde{I}_{n+1}=\tilde{I}_{n}+g_{k} 4 \pi \cdot \tilde{I}_{n}^{k} \sin \left[2 k(2 \pi n \delta)-\vartheta_{n}\right]$

The described above correlation function can be applied for analysis of the results of computer simulations in different accelerating and focusing structures. Simulations give usually particles trajectories as some discrete sequence of coordinates of particles in phase space $x_{n}=f\left(I_{n}, \vartheta_{n}, \tau\right)$. In this case $x_{n}$ can represent any coordinate of phase space. It is the most convenient to choose as step of sequence integer number of focusing periods of structure $\left(\tau_{n+1}=\tau_{n}+n\right)$. It corresponds to averaged over focusing period trajectories of particles. If initial conditions corresponds to the case $I_{n+1}=I_{n}$ and $\vartheta_{n+1}=\vartheta_{n}$ (zero current matched beam) the expression (4) becomes a sinusoid with constant amplitude and initial phase shift $\vartheta_{0}$. The appearance of space charge forces leads to determined by (8) amplitude and frequency modulation of trajectories. Modulation rate is determined by mapping (8).

The spectra of correlation function of particle trajectories are shown in fig. 1. The trajectories are the result of simulations of the beam dynamics in the test focusing channel. This test channel corresponds to the intermediate part of the linac for nuclear waste transmutation proposed in [6]. The parameters of the channel and beam are the following:

Type of focusing structure

Frequency of the accelerating field

Relative velocity of the particles

Normalized input emittance

Phase advance

The represented in fig. 1 results have been obtained by simulations with constant longitudinal particle velocities (no
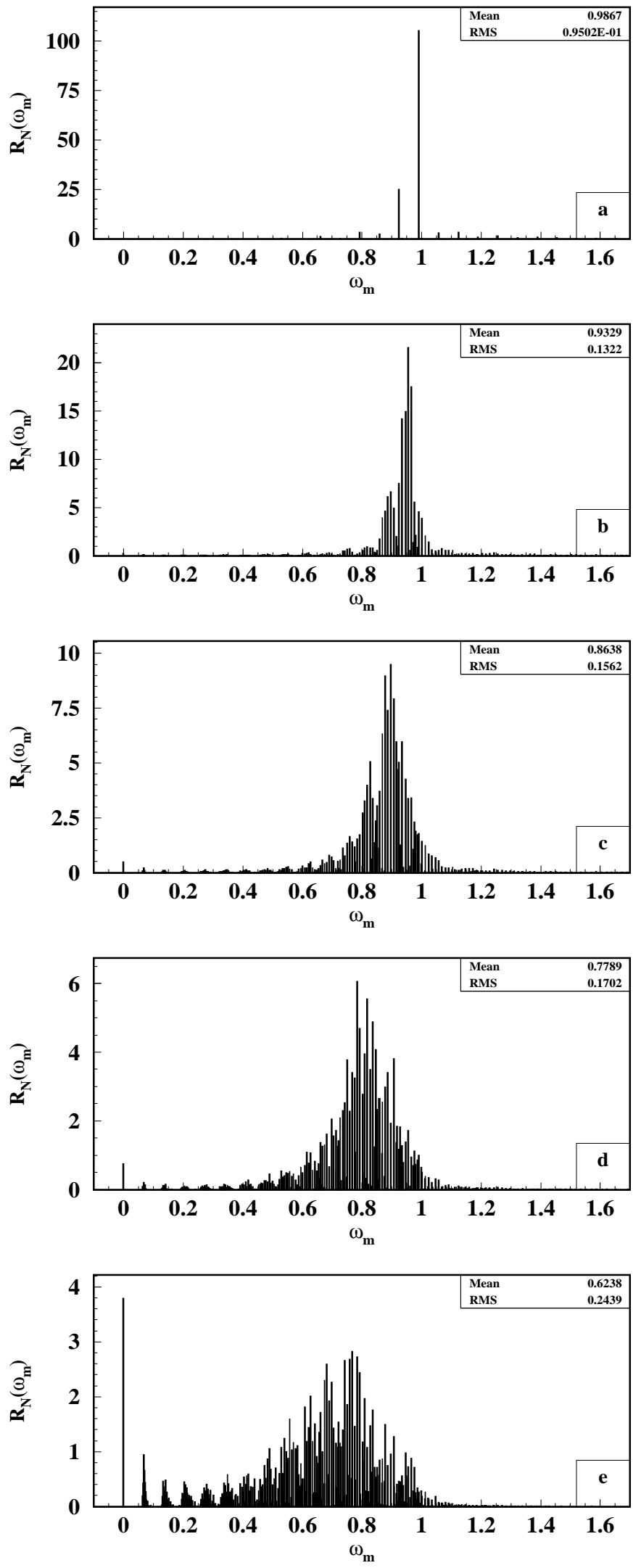

Figure 1. Spectra of correlation function $R_{N}\left(\omega_{m}\right)$ calculated for particle trajectories from test channel simulations at $h=0$ (a), $h=0.05$ (b), $h=0.124$ (c), $h=0.248$ (d), $h=0.496$ (e) 
acceleration) and for space charge forces calculated only for transverse planes. The simulations were carried out by means written in ITEP code „PROTON“ [7]. Test channel consists of 100 focusing periods and only 300 particles were used. The spectral densities of the correlation function in fig. 1 have been calculated for the Coulomb parameter $h=0,0.05,0.124$, $0.248,0.496$. The frequencies of the correlation function $\omega_{m}$ coincide in our case with phase advances at focusing period.

Fig. 1 shows that mean value of phase advance decreases due to linear shift and spectra width increases due to phase advance modulation with increasing of parameter $h$. The most noticeable feature of the spectra is the appearance of the central peak even for very small value of Coulomb parameter. This effect testifies that even for low Coulomb parameter there is the noticeable region in phase space where the behavior of the trajectories becomes chaotic.

The linear shift of phase advance depending on $h$ obtained from spectra is in a good accordance with analytical formula (6) as it is shown in fig.2.

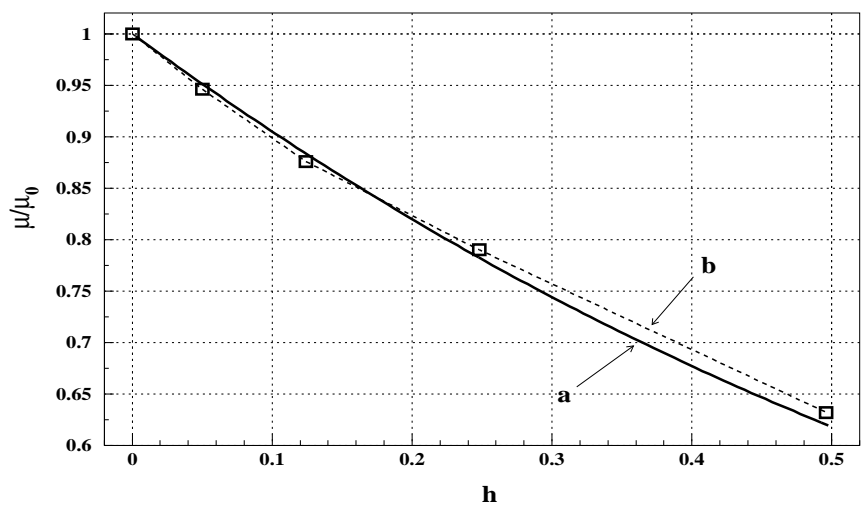

Figure 2. The phase advance dependence on $h$. a - calculated by formula (6), $\mathrm{b}$ - mean values of the spectra of correlation function $R_{N}\left(\omega_{m}\right)$.

The analysis of the results shows that there is considerable range of the parameter $h$ where simulations did not show any emittance growth. Nevertheless the relative power of the central peak significantly increases with $h$. This value allows to evaluate a relative size of regions of phase space with stochastic movement of particles. By assuming that this regions are responsible for beam halo appearance, the relative central peak power can be considered as a useful tool for comparison of different accelerating and focusing structures in terms of low level particle losses.

The dependence of central peak relative power and emittance growth in the test channel on $h$ is shown in fig.3. It can be seen that there is no threshold for appearance of the central peak whereas the emittance growth begins at $h>0.24$. When comparing the emittance growth curve with spectra, one can notice that at $h$ corresponding to the beginning of the rapid emittance growth, the low frequency border of the spectrum approaches to zero.

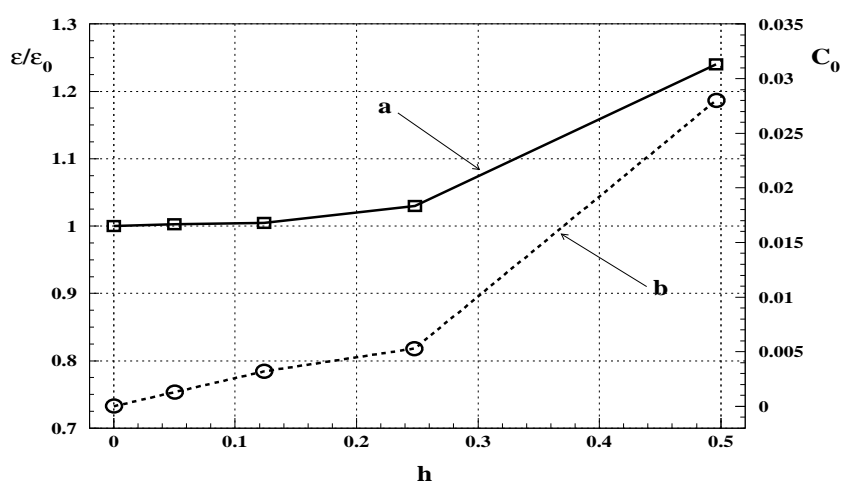

Figure 3. The dependence of the emittance growth (a) and the central peak relative power (b) on $h$.

\section{CONCLUSION}

The use of the correlation function is relatively simple procedure for analysis of spectral properties of particle trajectories calculated by simulations of beam dynamics in linacs. It does not need a big number of particles and can be used for the relatively short structures. This procedure allows to evaluate the influence of different nonlinear effects on beam dynamics. The relative measure of phase space regions where dynamics becomes stochastic can be estimated. It can be useful for the analysis of low level particle losses in different types of accelerating and focusing structures.

\section{REFERENCES}

[1] R. A. Jameson, „,Self-Consistent Beam Halo Studies \& Halo Diagnostic Development in a Continuous Linear Focusing Channel“, Los-Alamos Report LA-UR-94-3753.

[2] G. M. Zaslavskii, R. Z. Sagdeev, „Introduction to Nonlinear Physics“, „Nauka“, Moscow, 1988.

[3] V. V. Beloshapkin, G. M. Zaslavskii, „On The Spectral Properties of Dynamical Systems in The Transition Region from Order to Chaos", Physics Letters, v.97a, $\mathrm{N}^{\circ} 4,1983$.

[4] I. M. Kapchinskiy, „Theory of Linear Resonant Accelerators“, Moscow, „Energoizdat“", 1982.

[5] P. R. Zenkevich, A. P. Korolev, „Coulomb Structural Resonances in High Current Channels“, Preprint ITEP $\mathrm{N}^{\circ} 10$, Moscow, 1988.

[6] I. M. Kapchinskiy et al., „Linear Accelerator for Transmutation of Nuclear Power Production Wastes“, Preprint ITEP 100-92, Moscow, 1992.

[7] Vorobjev et al., Beam Dynamics Simulations in ITEP RFQ“, Preprint ITEP-52, Moscow, 1986. 